\title{
The impact of posttraumatic stress disorder on the symptomatology of borderline personality disorder
}

\author{
Sylvia Cackowski ${ }^{1 *}$, Tamar Neubauer $^{1}$ and Nikolaus Kleindienst ${ }^{2}$
}

\begin{abstract}
Background: Previous findings on the impact of co-occurring posttraumatic stress disorder (PTSD) in patients with borderline personality disorder (BPD) have revealed inconsistencies, which may have been related to small sample sizes or differences in the presence of childhood sexual abuse (CSA). In this study, the potentially aggravating impact of PTSD and the role of CSA were examined in a large cohort of BPD patients.

Methods: BPD patients with current PTSD $(n=142)$ were compared to BPD patients without PTSD $(n=225)$ regarding different BPD features such as non-suicidal self-injury. Further, we examined the potentially confounding role of CSA.

Results: BPD patients with PTSD showed elevated affect dysregulation, intrusions, dissociation, history of suicide attempts and self-mutilation compared to those with only BPD. The effects of PTSD on BPD patients regarding dissociation and the history of suicide attempts were at least partially related to CSA.

Conclusions: The additional diagnosis of PTSD in BPD patients can aggravate some, but not all BPD features. With respect to dissociation and suicide attempts, at least some of the impact seems to relate to CSA.
\end{abstract}

Keywords: Borderline personality disorder, Posttraumatic stress disorder, Childhood sexual abuse, Comorbidity

\section{Background}

Borderline personality disorder (BPD) shows high comorbidity rates with several psychiatric disorders [1-4]. The rates of co-occurring posttraumatic stress disorder (PTSD) within samples of BPD patients range between $30 \%$ and $79 \%$, with somewhat higher rates of comorbidity in clinical populations as compared to field-studies [3-7]. However, findings on the impact of additional PTSD on symptomatology in BPD patients remain inconsistent. Several studies revealed that women with BPD and cooccurring PTSD had worse general functioning, more severe BPD symptomatology [8-10], more frequent hospitalizations $[8,9,11]$, increased dissociation [9], suicidality and impulsivity $[8,12]$, as well as increased self-mutilating behaviour $[13,14]$. In a sample of 94

\footnotetext{
* Correspondence: sylvia.cackowski@zi-mannheim.de

${ }^{1}$ Department of Psychosomatic Medicine and Psychotherapy, Central

Institute of Mental Health, Medical Faculty Mannheim, Heidelberg University,

Mannheim, Germany

Full list of author information is available at the end of the article
}

BPD patients, Harned and colleagues found higher rates of self-injury and suicide attempts based on interpersonal reasons in BPD patients with co-occurring PTSD, as well as more pronounced emotion dysregulation [13]. However, there were no differences found in the number of met BPD criteria and general psychosocial functioning between BPD patients with or without PTSD. In a longitudinal study by Gunderson and colleagues, co-occurring PTSD in BPD patients was associated with poorer 2-year outcomes based on the Global Assessment of Functioning Scale (GAF) $[15,16]$. Furthermore, a 10-year follow-up study showed that the likelihood of symptom remission in BPD patients was lower in those with co-occurring PTSD [17]. Previous studies also revealed that the reported extent of sexual $[4,8,13]$, physical, verbal, and emotional abuse $[3,8]$ was higher in patients with a combined diagnosis of BPD and PTSD. Nonetheless, several other studies did not find any significant impact of co-occurring 
PTSD on BPD symptom severity [11, 13, 18], suicidality, hospitalizations [13], or physical, emotional and sexual abuse severity [9].

Independent of ones BPD or PTDS diagnosis, childhood sexual abuse (CSA) has been found to relate to serious aspects such as self-mutilation, suicidality and dissociation $[19,20]$. Although some controversial results have pointed out that CSA is neither a necessary nor sufficient factor in the development of BPD [19, 21-23], CSA has been intensely discussed as an etiological factor for BPD $[14,18]$. Several studies have shown that the severity of CSA predicts the severity of BPD symptomatology $[2,3,21,24,25]$. It has been hypothesized that CSA may explain the presence and impact of PTSD in BPD patients, i.e., that CSA, rather than a PTSD diagnosis, might be predictive of self-mutilating behaviour, as well as more severe symptomatology in patients with BPD [13, 18].

Previous research addressing the relationship between BPD, PTSD and CSA has some noteworthy limitations. As Nepon and colleagues [26] noticed, samples of highly symptomatic treatment seeking patients, as studied by Harned and colleagues [13], may not be representative of a broader BPD population. On the other hand, it should also be noted that research on treatment seeking patients is particularly relevant for therapists, who seek information about the complexity of conditions of their clientele. Furthermore there is the assumption that discrepancies between the results of Harned and colleagues [13] and other (epidemiologic) studies [4] might be a consequence of different sample sizes. Although the patients investigated in our study were also treatment seeking, we aimed to overcome the limitation of a small sample size. To further enhance representability of our sample, no specific inclusion criteria were stated concerning the presence or frequency of self-injury and suicide attempts.

We aimed to investigate a large clinical sample of BPD patients divided in subgroups of patients with versus without co-occurring PTSD, to clarify whether additional PTSD is related to major aspects of BPD symptomatology such as the number of fulfilled BPD criteria, as well as suicidal and self-harming behaviour. In a next step, we analysed the impact of CSA. This seems important, as CSA affects BPD pathology [2, 3, 21], but does not necessarily lead to a PTSD diagnosis [27]. For instance, we expect CSA (rather than PTSD) to be predictive of self-mutilating behaviour and general symptom severity $[13,18]$.

\section{Methods}

\section{Sample}

Participants were treatment-seeking patients, mostly for a presumed diagnosis of BPD. Criteria for inclusion in the study were female gender, age between 18-65 years and a diagnosis of BPD according to DSM-IV [28]. Participants were excluded from the study if they met life-time criteria for schizophrenia, bipolar-I disorder, or mental disorder due to brain damage. All diagnostic and observer based assessment instruments were conducted by trained psychologists and physicians. BPD diagnosis (according to DSM-IV criteria) was determined by using the International Personality Disorder Examination (IPDE) [29]. Apart from the number of met BPD criteria, a dimensional IPDE score was calculated. Axis-I diagnoses, including PTSD were assessed using the Structural Clinical Interview for DSM-IV (SCID-I) [15]. Besides assessing whether the diagnostic criteria for PTSD was currently met (current PTSD), we also assessed whether PTSD criteria was met in the past. Up to $84 \%$ of the patients with lifetime PTSD met the criteria for a current PTSD, therefore analyses were based on the current PTSD diagnosis, which was free of missing values (the results were almost identical when based on current or lifetime PTSD). A total of 722 female patients were assessed for eligibility, whereby 355 (49.2\%) of them were not included in the study, as not all of the predefined criteria for participation in the study were met. All of the remaining 367 female patients were included in the study.

\section{Assessment of clinical characteristics}

To quantify borderline-typical symptomatology, the Borderline Symptoms List- 95 (BSL) [30] was applied, which is a self-report scale consisting of seven subscales (self-perception, affect regulation, auto aggression, dysphoria, isolation, intrusions, and hostility) and a general severity score of BPD symptomatology. The psychometric criteria of the BSL have been investigated in several studies and have demonstrated good reliability and validity [30]. Further clinical assessments comprised questionnaires on trait dissociation measured by the Dissociative Experiences Scale (DES) [31], childhood trauma history measured by Childhood Trauma-Questionnaire (CTQ) [32] and an assessment of the presence and frequency of previous suicide attempts, as well as the history of self-mutilation ("Have you ever had a suicide attempt in your life? How many suicide attempts did you have in your life? Have you ever used self-injuring behaviour?"). Participants were informed about the definition of self-injuring behaviour, which was determined as "any deliberate and self-inflicted behaviour with the intention to harm or destroy body tissue without suicidal intent".

\section{Procedure}

The current study is part of an observational study which was conducted from April 2001 to October 2007 
at two German Departments of Psychiatry specialized in treatment of BPD: the Department of Psychiatry of the University in Freiburg and the Central Institute of Mental Health in Mannheim. The patients included in the study were treatment-seeking patients, mostly for a presumed diagnosis of BPD. Definite BPD diagnosis (according to DSM-IV; [28]) was determined by use of the International Personality Disorder Examination (IPDE; [29]). Axis-I diagnoses were established by use of the structural clinical interview for DSM-IV (SCID-I; [15]). The following inclusion criteria were required for study entry:

- female gender

- age ranging from 18 to 65

- established diagnosis of BPD (according to DSM-IV) lifetime

- written informed consent.

Patients with a lifetime diagnosis of schizophrenia, bipolar I disorder, or mental disorders due to brain damage were not included in this study. The investigation was conducted in accordance with GCP guidelines and the declaration of Helsinki and was approved by the Ethics committee of the Medical Faculty of Heidelberg University. The procedure of the study was explained in detail and a written informed consent was signed by all participants. Data on the potentially aggravating impact of attention-deficit hyperactivity disorder for BPD have been published elsewhere [33].

\section{Statistical analysis}

Regression models were used to test whether a diagnosis of current PTSD was related to continuous data (such as DES total score). For modelling, binary data logistic regression was applied. Chi-square tests were used to compare categorical variables such as education, employment status and marital status. To compare ordinal data, such as years of schooling across groups, the Kolmogorov-Smirnoff test was used. We indicated with an asterisk which variables would stay significant on the alpha level of 0.05 (2-tailed) after Bonferroni correction.

To examine whether the relation between PTSD and BPD psychopathology (BSL, DES, suicidal and self-harming behaviour) was associated with CSA (measured by CTQ), we applied a mediational model as described by Baron and Kenny [34]. When these analyses referred to continuous dependent variables, regression coefficient relating PTSD and BPD-psychopathology was decomposed into two additive components: (a) the indirect effect related to CSA, and (b) the regression coefficient after controlling for CSA. These two components enabled us to determine to which extent the connection between PTSD and a dependent variable (BSL, DES, suicidal and self-harming behaviour) was associated with CSA [35]. As pointed out by Preacher and Hayes [36], the formal evaluation of statistical significance of the indirect effect should be based on non-parametric methods, as the sampling distribution of the indirect effect is typically skewed. This skewedness violates the assumption of normality, which is at the base of e.g., the Sobel-test. We followed the recommendation by Hayes [37] and estimated the standard error of the parameter corresponding to the indirect effect from bootstrapping using 10,000 bootstrapping samples for each analysis by applying the macro PROCESS realized by Hayes in SPSS [37]. The parameters corresponding to the indirect effect were considered significant if the 95\% confidence interval around the point estimate of the parameter did not include zero.

\section{Results}

\section{Demographic and clinical characteristics}

Of the 367 BPD patients, 142 (38.7\%) met the criteria for current PTSD. Another 27 patients met the criteria for lifetime PTSD but not current PTSD. The most commonly reported traumatic events were ${ }^{1}$ : sexual abuse or harassment $(76 \%)$, physical violence $(35 \%)$, witness of traumatic events such as violence against or abuse of others $(28 \%)$, accidents (7\%) and raid or threat with a weapon (5\%). More than one trauma type was experienced by $54 \%$ of these patients.

Patients with and without PTSD did not differ significantly on any demographic characteristics (see Table 1). However, self-reported CSA occurred more frequently in the subgroup of BPD patients with a co-occurring diagnosis of PTSD than in BPD patients without cooccurring PTSD $(81.2 \%$ vs. $53.1 \%, p \leq 0.01)$. The correlation between self-reported CSA and a diagnosis of PTSD was $0.429(p \leq 0.01)$. Further clinical characteristics of BPD patients with and without PTSD and statistical group comparisons are depicted in Table 2.

\section{The role of CSA}

As shown in the Table 3, dissociation as assessed by the DES total scores was significantly related to the diagnosis of PTSD $(p=0.02)$. When one separates the regression coefficient $(b=5.40)$ into an indirect component related to CSA $(\mathrm{b}=4.16, \mathrm{p} \leq 0.01)$ and the direct component after controlling for CSA $(b=1.24, p=0.62)$, the results suggest that the relationship between the diagnosis of PTSD and higher DES scores is essentially related to the presence of CSA $(p \leq 0.01)$ and is no longer significant after controlling for CSA. Similarly, we detected a significant relationship between PTSD and the occurrence of at least one suicide attempt $(p \leq 0.01)$. In percentages, $79.5 \%$ of BPD patients with co-occurring PTSD tried to commit suicide, compared to $66.8 \%$ of 
Table 1 Demographic characteristics of BPD patients with and without PTSD

\begin{tabular}{|c|c|c|c|c|c|c|c|}
\hline & Total & & PTSD abse & & PTSD prese & & $P$ \\
\hline & $\mathrm{N}$ & $\%$ & $\mathrm{~N}$ & $\%$ & $\mathrm{~N}$ & $\%$ & \\
\hline Age $(M \pm S D)$ & $28.6 \pm 7.9$ & & $28.8 \pm 7.9$ & & $28.1 \pm 7.9$ & & 0.46 \\
\hline Schooling & & & & & & & 0.08 \\
\hline No schooling & 5 & 1.5 & 3 & 1.5 & 2 & 1.5 & \\
\hline 9 years of schooling & 73 & 21.4 & 42 & 20.6 & 31 & 22.6 & \\
\hline 10 years of schooling & 150 & 44.0 & 80 & 39.2 & 70 & 51.1 & \\
\hline 12-13 years of schooling & 113 & 33.1 & 79 & 38.7 & 34 & 24.8 & \\
\hline Education & & & & & & & 0.13 \\
\hline Apprenticeship, University & 205 & 61.0 & 128 & 64.3 & 77 & 56.2 & \\
\hline No degree & 131 & 39.0 & 71 & 35.7 & 60 & 43.8 & \\
\hline Employment status & & & & & & & 0.48 \\
\hline Employed & 86 & 25.2 & 54 & 26.6 & 32 & 23.2 & \\
\hline Unemployed & 255 & 74.8 & 149 & 73.4 & 106 & 76.8 & \\
\hline Marital status & & & & & & & 0.41 \\
\hline Single, divorced/widowed & 273 & 82.2 & 164 & 83.7 & 109 & 80.1 & \\
\hline Married & 59 & 17.8 & 32 & 16.3 & 27 & 19.9 & \\
\hline
\end{tabular}

Valid numbers and percentages (i.e., not including missing data) are presented

Table 2 Clinical characteristics of BPD patients with and without PTSD

\begin{tabular}{|c|c|c|c|c|}
\hline & Total & PTSD absent & PTSD present & $P$ \\
\hline & $M+/-S D$ & $M+/-S D$ & $M+/-S D$ & \\
\hline \multicolumn{5}{|l|}{ CTQ } \\
\hline History of CSA & $63.3 \%$ & $53.1 \%$ & $81.2 \%$ & $\leq 0.01$ \\
\hline DES & $29.74+/-16.19$ & $27.37+/-15.37$ & $33.47+/-16.81$ & $\leq 0.01$ \\
\hline \multicolumn{5}{|l|}{ Suicide attempts/self-mutilation } \\
\hline Frequency of suicide attempts & $4.14+/-7.41$ & $4.26+/-8.50$ & $3.98+/-5.51$ & 0.90 \\
\hline History of suicide attempts & $71.92 \%$ & $66.8 \%$ & $79.5 \%$ & 0.02 \\
\hline History of self-mutilation & $95.02 \%$ & $92.7 \%$ & $98.4 \%$ & 0.03 \\
\hline \multicolumn{5}{|l|}{ BSL } \\
\hline BPD severity & $206.41+/-68.30$ & $201.03+/-70.87$ & $215.12+/-63.23$ & 0.07 \\
\hline Self-perception & $35.65+/-17.83$ & $34.42+/-18.50$ & $37.64+/-16.57$ & 0.12 \\
\hline Affect regulation & $32.59+/-10.45$ & $31.68+/-10.98$ & $34.09+/-9.39$ & 0.04 \\
\hline Auto aggression & $28.69+/-13.07$ & $27.77+/-13.47$ & $30.17+/-12.32$ & 0.11 \\
\hline Dysphoria & $32.65+/-5.94$ & $32.58+/-6.24$ & $32.77+/-5.44$ & 0.78 \\
\hline Isolation & $23.57+/-10.51$ & $23.36+/-10.59$ & $23.90+/-10.40$ & 0.65 \\
\hline Intrusions & $13.86+/-8.19$ & $12.49+/-8.11$ & $16.05+/-7.86$ & $\leq 0.001$ \\
\hline Hostility & $10.15+/-5.22$ & $10.04+/-5.35$ & $10.32+/-5.03$ & 0.64 \\
\hline \multicolumn{5}{|l|}{ IPDE } \\
\hline Number of BPD criteria & $6.63+/-1.24$ & $6.55+/-2.02$ & $6.76+/-1.19$ & 0.90 \\
\hline BPD dimensional score & $14.49+/-1.97$ & $14.39+/-1.26$ & $14.66+/-1.88$ & 0.21 \\
\hline
\end{tabular}


Table 3 Indirect effects related to CSA

\begin{tabular}{llll}
\hline & $\begin{array}{l}\text { Total effect of PTSD } \\
\text { Regression coefficients, } \\
\text { SEs and } P \text {-values }\end{array}$ & $\begin{array}{l}\text { Indirect component related to CSA } \\
\text { Regression coefficients, } \\
\text { SEs and } P \text {-values }\end{array}$ & $\begin{array}{l}\text { Direct component after controlling for CSA } \\
\text { Regression coefficients, } \\
\text { SEs and } P \text {-values }\end{array}$ \\
\hline $\begin{array}{l}\text { DES } \\
\begin{array}{l}\text { Suicide attempts/self-mutilation } \\
\text { History of suicide attempts** }\end{array}\end{array}$ & $\begin{array}{l}5.40 \pm 2.32(p=0.02) \\
\text { BSL }\end{array}$ & $4.16 \pm 1.38(p \leq 0.01)^{*}$ & $1.24 \pm 2.51(p=0.62)$ \\
$\quad \begin{array}{l}\text { Affect regulation } \\
\text { Intrusions }\end{array}$ & $3.53 \pm 1.40(p=0.01)$ & $1.11 \pm 0.63(p=0.08)$ & $0.40 \pm 0.33(p=0.23)$ \\
\hline
\end{tabular}

"Remains statistically significant after Bonferroni-correction

${ }^{* *}$ Remains statistically significant when considering lifetime PTSD $(p \leq 0.01)^{*}$

BPD patients without PTSD. CSA significantly affected the link between PTSD and the history of suicide attempts in BPD patients, as this link became nonsignificant after controlling for CSA $(p=0.23)$.

As the (lifetime) history of at least one suicide attempt might be related to lifetime PTSD rather than to current PTSD, analyses were also carried out for lifetime PTSD. Hereby, the results were fully confirmed. In contrast to the results on the occurrence of at least one suicide attempt, the frequency of previous suicide attempts was not significantly related to the diagnosis of PTSD $(p=0.90)$. Concerning the history of self-mutilation, the relation with PTSD diagnosis showed a trend $(p=0.09)$. 98.4\% of BPD patients with PTSD self-mutilated compared to $92.7 \%$ of BPD patients without PTSD. There was no significant effect of CSA on self-mutilation and the connection stayed non-significant after controlling for CSA $(p=0.10)$. As further shown in Table 3, two of the seven BSL subscales showed a significant relation with PTSD diagnosis: intrusions $(p \leq 0.01)$ and affect regulation $(p=0.01)$.

\section{Discussion}

This study adds to a growing body of evidence about patients with BPD and co-occurring PTSD. According to our results, PTSD is significantly related to dissociation (DES) and a history of suicide attempts in BPD patients. A trend was found for the impact of PTSD on the symptom severity (BSL) and the history of self-mutilation in patients with BPD.

Our finding that patients with co-occurring current PTSD scored significantly higher on the DES than patients without a co-occurring diagnosis, is in line with the findings from Heffernan and Cloitre, who also reported higher scores on the DES in the group of BPD patients with co-occurring PTSD [9]. However, this association became non-significant in our study after controlling for CSA, suggesting that the effect of PTSD on dissociation in BPD patients may be associated with the history of sexual abuse in childhood [38, 39]. Our finding that significantly more BPD patients with PTSD reported having attempted suicide than patients without PTSD diagnosis is consistent with the results of several previous studies $[4,8]$. When controlling for CSA, the connection between PTSD and the history of suicide attempts disappeared, suggesting that CSA and not PTSD, is influencing suicidality rates in BPD patients. Similar previous results have been reported stating that severity of CSA is associated with higher rates of suicide attempts [19, 20]. For example, Ferraz and colleagues revealed that in BPD patients, the presence, number and severity of previous suicide attempts are significantly predicted by CSA [40]. In recent studies $[8,13]$ it has been assumed that CSA could be associated with the effects of PTSD in BPD patients and even account for the presence of PTSD symptoms among patients with BPD [18]. Interestingly, BPD patients with and without PTSD did not differ in terms of the frequency of suicide attempts. However, our findings indicate that the frequency of suicide attempts was significantly related to CSA.

While self-mutilation tended to be higher in BPD patients with co-occurring PTSD, this trend was not statistically significant, despite the relatively large number of patients included in our study. This is not fully consistent with previous studies, which revealed that BPD patients with co-occurring PTSD had a higher frequency of intentional self-injury, typically being triggered by flashbacks, nightmares or thoughts about sexual abuse or rape $[13,14]$. The slight differences between our finding and previous results might relate to the very high prevalence of self-mutilation in our study ( $>90 \%$ in both subsamples of BPD patients with and without PTSD), which might have caused a ceiling effect. Self-mutilation was unrelated to CSA in our sample.

With respect to the impact of PTSD on BPD severity, our study provided preliminary evidence that some specific aspects (rather than overall severity) might be impacted by co-occurring PTSD. With respect to overall severity, we only found a non-significant trend towards 
higher total scores in the BSL. We found no evidence for higher scores with respect to the number of BPD criteria and the dimensional IPDE score in the subgroup of patients with co-occurring PTSD. This is in line with previous reports that PTSD does not necessarily affect the extent of BPD psychopathology $[11,13,18]$. With respect to the facets of psychopathology (BSL), we did, however, detect higher scores in the subscales "intrusion" and "affect regulation" in BPD patients with co-occurring PTSD. This indicates that patients with both diagnoses report more intrusions and elevated problems in affect regulation than BPD patients without a co-occurring PTSD diagnosis. Overall, these results are in line with the findings by Harned and colleagues, who did not find an effect for PTSD on the number of BPD criteria and the dimensional score, but on emotion regulation [13]. However, these results do not support the outcomes of other studies, which indicated that co-occurring PTSD has a negative effect on general BPD symptomatology $[8,10,14]$.

Our study has both strengths and limitations. A strength of our study is the relatively large sample of well diagnosed participants. All patients underwent standardized diagnostics including structured interviews for BPD (IPDE) and Axis-I disorders (SCID-I), which were conducted by experienced diagnosticians. With respect to the limitations, we would like to emphasize that our sample only included treatment-seeking female BPD patients recruited at specialized university settings. Hence, generalizations beyond the population investigated in this study should be made with caution. Furthermore, as most of our dependent variables were based on retrospective selfreports, there is a possibility of bias. One significant limitation concerns the assessment of the presence and frequency of previous suicide attempts and the history of self-mutilation in this study. As we did not use a wellestablished and validated measure, the validity of our results on suicide attempts and self-mutilation might be constrained. Future studies investigating this topic should make use of measures such as the Columbia-Suicide Severity Rating Scale (C-SSRS), which have been developed to consistently define and classify these behaviours and show good psychometric properties [41]. Furthermore, as we conducted an observational study, the possibility of making any causal conclusions regarding the impact of PTSD and CSA in BPD patients is precluded. Finally, multiple testing poses the question regarding the inflation of alpha-error. To address this issue, a Bonferroni-correction was applied. We found that dissociation and a history of at least one suicide attempt would still hold after correction for multiple testing.

\section{Conclusions}

Despite these limitations, the results of our study should be considered in further research, as well as in clinical practice. From an etiological perspective, it is interesting that both dissociation and a history of at least one suicide attempt were related to CSA rather than to PTSD. From a clinical perspective, our data highlights the need to address these aspects in the treatment of BPD patients with co-occurring PTSD or a history of CSA, as suicide attempts are highly prevalent within this population of patients and as dissociation has been shown to detrimentally affect the success of treatment in both BPD [13, 14] and PTSD patients [3].

\section{Endnote}

${ }^{1}$ The mentioned percentages refer to 93 of the 142 patients with PTSD. For the remaining 49 patients information regarding the type of trauma (from SCID-I) was either missing or not entirely conclusive retrospectively.

\section{Abbreviations}

$\mathrm{BPD}$, borderline personality disorder; BSL, borderline symptoms list- 95; CSA, childhood sexual abuse; C-SSRS, Columbia-Suicide Severity Rating Scale; $C T Q$, childhood trauma-questionnaire; DES, dissociative experiences scale; GAF, global assessment of functioning scale; IPDE, International Personality Disorder Examination; PTSD, posttraumatic stress disorder; SCID-I, structural clinical interview for DSM-IV; SPSS, statistical package for the social sciences

\section{Acknowledgements}

We thank all participants for their collaboration in this study. We also thank Melanie Harned for previous research in this field and the inspiration for our investigation.

\section{Funding}

The study has been funded by the Borderline Personality Disorder Research Foundation, New York.

\section{Availability of data and materials}

The dataset supporting the conclusions of this article is available on request to Nikolaus Kleindienst (Nikolaus.kleindienst@zi-mannheim.de).

\section{Authors' contributions}

NK drafted the manuscript, conducted the analysis and led the interpretation of the data. SC made substantial contributions to the manuscript conceptualising and revision. TN assisted to in the manuscript drafting. All authors approved the final manuscript.

\section{Competing interests}

The authors declare that they have no competing interests.

Consent for publication

Not applicable.

\section{Ethics approval and consent to participate}

The investigation was conducted in accordance with GCP guidelines and the declaration of Helsinki and was approved by the Ethics committee of the Medical Faculty of Heidelberg University. The procedure of the study was explained in detail and a written informed consent was signed by all participants.

\section{Author details}

${ }^{1}$ Department of Psychosomatic Medicine and Psychotherapy, Central Institute of Mental Health, Medical Faculty Mannheim, Heidelberg University, Mannheim, Germany. ${ }^{2}$ Institute of Psychiatric and Psychosomatic

Psychotherapy, Central Institute of Mental Health, Medical Faculty Mannheim, Heidelberg University, Mannheim, Germany.

Received: 17 December 2015 Accepted: 12 July 2016

Published online: 01 August 2016 


\section{References}

1. Grant BF, Chou SP, Goldstein RB, Huang B, Stinson FS, Saha TD, et al. Prevalence, correlates, disability, and comorbidity of DSM-IV borderline personality disorder: results from the Wave 2 National Epidemiologic Survey on Alcohol and Related Conditions. J Clin Psychiatry. 2008;69:533-45.

2. Zanarini MC, Frankenburg FR, Hennen J, Reich DB, Silk KR. Axis I comorbidity in patients with borderline personality disorder: 6-year follow-up and prediction of time to remission. Am J Psychiatry. 2004;161:2108-14.

3. Zanarini MC, Frankenburg FR, Dubo ED, Sickel AE, Trikha A, Levin A, et al Axis II comorbidity of borderline personality disorder. Compr Psychiatry. 1998:39:296-302.

4. Pagura J, Stein MB, Bolton JM, Cox BJ, Grant B, Sareen J. Comorbidity of borderline personality disorder and posttraumatic stress disorder in the U.S. population. J Psychiatric Res. 2010;44:1190-8.

5. Zimmerman M, Mattia Jl. Axis I Diagnostic Comorbidity and Borderline Personality Disorder. Compr Psychiatry. 2003;40:245-52

6. Westphal M, Olfson M, Bravova M, Gameroff MJ, Gross R, Wickramaratne P, et al. Borderline personality disorder, exposure to interpersonal trauma, and psychiatric comorbidity in urban primary care patients. Psychiatry. 2013;76:365-80.

7. Frías Á, Palma C. Comorbidity between Post-Traumatic Stress Disorder and Borderline Personality Disorder: A Review. Psychopathology. 2015;48:1-10.

8. Zlotnick C, Johnson DM, Yen S, Battle CL, Sanislow CA, Skodol AE, et al. Clinical features and impairment in women with borderline personality disorder (BPD) with posttraumatic stress disorder (PTSD), BPD without PTSD, and other personality disorders with PTSD. J Nerv Ment Dis. 2003;191:706-13.

9. Heffernan K, Cloitre M. A comparison of posttraumatic stress disorder with and without borderline personality disorder among women with a history of childhood sexual abuse: etiological and clinical characteristics. J Nerv Ment Dis. 2000;188:589-95

10. Rüsch N, Corrigan PW, Bohus M, Kühler T, Jacob GA, Lieb K. The impact of posttraumatic stress disorder on dysfunctional implicit and explicit emotions among women with borderline personality disorder. J Nerv Ment Dis. 2007;195:537-9.

11. Bolton EE, Mueser KT, Rosenberg SD. Symptom correlates of posttraumatic stress disorder in clients with borderline personality disorder. Compr Psychiatry. 2006:47:357-61.

12. Spokas M, Wenzel A, Stirman SW, Brown GK, Beck AT. Suicide risk factors and mediators between childhood sexual abuse and suicide ideation among male and female suicide attempters. J Trauma Stress. 2009;22:467-70.

13. Harned MS, Rizvi SL, Linehan MM. Impact of co-occurring posttraumatic stress disorder on suicidal women with borderline personality disorder. Am J Psychiatry. 2010;167:1210-7.

14. Complex HJL, PTSD. A syndrome in survivors of prolonged and repeated trauma. J Trauma Stress. 1992:5:377-91.

15. First MB, Spitzer RL, Gibbon M, Williams JB. User's quide for the Structured clinical interview for DSM-IV axis I disorders SCID-I: clinician version. American Psychiatric Pub; 1997

16. Gunderson JG, Daversa MT, Grilo CM, McGlashan TH, Zanarini MC, Shea MT, et al. Predictors of 2-year outcome for patients with borderline personality disorder. Am J Psychiatry. 2006;163:822-6.

17. Zanarini MC, Frankenburg FR, Hennen J, Reich DB, Silk KR. Prediction of the 10-year course of borderline personality disorder. Am J Psychiatry. 2006;163:827-32

18. Zlotnick C, Franklin CL, Zimmerman M. Is Comorbidity of Posttraumatic Stress Disorder and Borderline Personality Disorder Related to Greater Pathology and Impairment? Am J Psychiatry. 2002;159:1940-3.

19. Browne A, Finkelhor D. Impact of child sexual abuse: A review of the research. Psychol Bull. 1986;99:66-77.

20. Polusny MA, Follette VM. Long-term correlates of child sexual abuse: Theory and review of the empirical literature. Appl Prev Psychol. 1995:4:143-66.

21. Paris J, Zweig-Frank H, Guzder J. The role of psychological risk factors in recovery from borderline personality disorder. Compr Psychiatry. 1993;34:410-3.

22. Fossati A, Madeddu F, Maffei C. Borderline Personality Disorder and childhood sexual abuse: a meta-analytic study. J Personal Disord. 1999;13:268-80.

23. Zanarini MC, Williams AA, Lewis RE, Reich RB, Vera SC, Marino MF, et al. Reported pathological childhood experiences associated with the development of borderline personality disorder. Am J Psychiatry. 1997;154:1101-6.
24. Silk KR, Lee S, Hill EM, Lohr NE. Borderline personality disorder symptoms and severity of sexual abuse. Am J Psychiatry. 1995;152:1059-64.

25. Hong PY, llardi SS, Lishner DA. The aftermath of trauma: The impact of perceived and anticipated invalidation of childhood sexual abuse on borderline symptomatology. Psychol Trauma Theory Res Practice Policy. 2011;3:360.

26. Nepon J, Pagura J, Sareen J. Study limitations in report of suicidal behavior among women with co-occurring PTSD and borderline personality disorder. Am J Psychiatry. 2011;168:328.

27. Kessler RC, Sonnega A, Bromet E, Hughes M, Nelson CB. Posttraumatic stress disorder in the National Comorbidity Survey. Arch Gen Psychiatry. 1995;52:1048-60.

28. American Psychiatric Association. Diagnostic and Statistical Manual of Mental Disorders: Text Revision (DSM-IV-TR). Washington: American Psychiatric Association; 2000.

29. Loranger AW, Sartorius N, Andreoli A, Berger P, Buchheim P, Channabasavanna SM, et al. The International Personality Disorder Examination: The World Health Organization/Alcohol, Drug Abuse, and Mental Health Administration international pilot study of personality disorders. Arch Gen Psychiatry. 1994;51:215-24.

30. Bohus M, Limberger MF, Frank U, Chapman AL, Kühler T, Stieglitz R-D. Psychometric properties of the borderline symptom list (BSL). Psychopathology. 2007:40:126-32.

31. Bernstein EM, Putnam FW. Development, reliability, and validity of a dissociation scale. J Nerv Ment Dis. 1986;174:727-35.

32. Bernstein DP, Stein JA, Newcomb MD, Walker E, Pogge D, Ahluvalia T, et al. Development and validation of a brief screening version of the Childhood Trauma Questionnaire. Child Abuse Negl. 2003;27:169-90.

33. Philipsen A, Limberger MF, Lieb K, Feige B, Kleindienst N, Ebner-Priemer U, et al. Attention-deficit hyperactivity disorder as a potentially aggravating factor in borderline personality disorder. Br J Psychiatry. 2008;192:118-23.

34. Baron RM, Kenny DA. The moderator-mediator variable distinction in social psychological research: Conceptual, strategic, and statistical considerations. J Pers Soc Psychol. 1986;51:1173-82.

35. Zhao X, Lynch JG, Chen Q. Reconsidering Baron and Kenny: Myths and truths about mediation analysis. J Consum Res. 2010;37:197-206.

36. Preacher KJ, Hayes AF. SPSS and SAS procedures for estimating indirect effects in simple mediation models. Behav Res Methods Instrum Comput. 2004;36:717-31.

37. Hayes AF. PROCESS: A versatile computational tool for observed variable mediation, moderation, and conditional process modeling [White paper]. 2012.

38. Brodsky BS, Cloitre M, Dulit RA. Relationship of dissociation to self-mutilation and childhood abuse in borderline personality disorder. Am J Psychiatry. 1995; 152:1788-92

39. Shearer SL. Dissociative phenomena in women with borderline personality disorder. Am J Psychiatry. 1994;151:1324-8.

40. Ferraz L, Portella MJ, Vallez M, Gutierrez F, Martin-Blanco A, Martin-Santos R, et al. Hostility and childhood sexual abuse as predictors of suicidal behaviour in Borderline Personality Disorder. Psychiatry Res. 2013;210:980-5.

41. Posner K, Brown GK, Stanley B, Brent DA, Yershova KV, Oquendo MA, et al. The Columbia-Suicide Severity Rating Scale: initial validity and internal consistency findings from three multisite studies with adolescents and adults. Am J Psychiatry. 2011;168:1266-77. 